\title{
Erratum
}

\section{A Constraint Programming Approach to Extract the Maximum Number of Non-Overlapping Test Forms}

DMITRY I. BELOV AND RONALD D. ARMSTRONG

Published online: 28 July 2006

The publisher regrets the following errors which appeared in the article "A Constraint Programming Approach to Extract the Maximum Number of Non-Overlapping Test Forms" by Dmitry I. Belov and Ronald D. Armstrong in Computational Optimization and Applications, volume 33, numbers 2/3, pages 319-332.

On page 323 in Section 5, Algorithm 1 (below) was inadvertently omitted and should have appeared above the paragraph which begins with the words "Since on Step 6...."

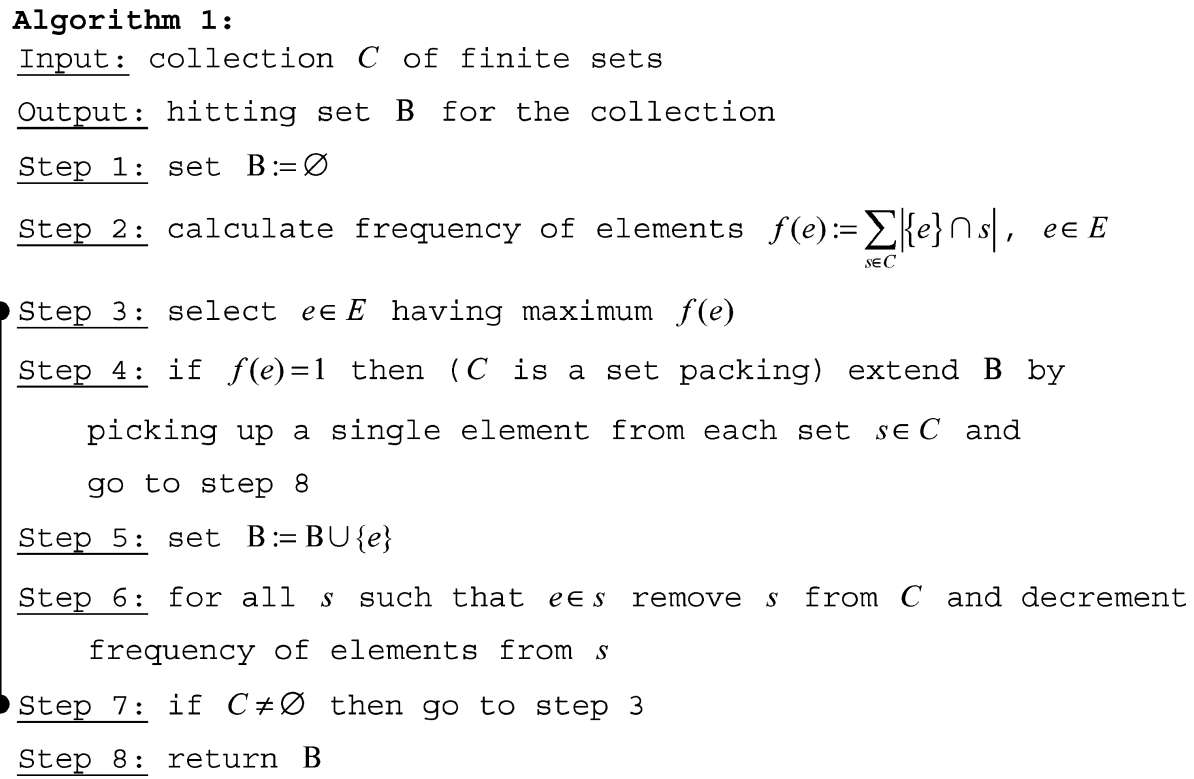


Also, on page 326 in Algorithm 3, the second ' $\mathrm{C}$ tilde' in the "Input" should actually be a ' $C$ '. Algorithm 3 should have appeared on page 325 at the gap between lines "...for the MSP." and "To find an initial lower bound...". The correct Algorithm 3 can be found below:

Algorithm 3:

Input: subcollection $\tilde{C} \subseteq C$

Output: set packing $Q \subseteq \tilde{C}$

Step 1: set $Q:=\varnothing$

Step 2: choose a set $s \in \tilde{C}$ with maximum $|N(s)|$
Step 3: set $Q:=Q \cup\{s\}$ and $\tilde{C}:=\tilde{C} \cap N(s)$
Step 4: if $\tilde{C} \neq \varnothing$ go to step 2
Step 5: return $Q$

The online version of the original article can be found at http://dx.doi.org/10.1007/s10589005-3058-z. 